\title{
Contribución del transporte aéreo a la conectividad territorial. El caso de Colombia
}

Óscar Díaz-Olariaga. Universidad Santo Tomás, Bogotá, Colombia.

RESUMEN | El presente artículo analiza la influencia y contribución del transporte aéreo a la conectividad territorial, tomando Colombia como caso de estudio. La invaluable contribución del transporte aéreo a la conectividad entre las diferentes regiones del país ha sido posible gracias al desarrollo de un conjunto de políticas públicas dirigidas a tal industria, implementadas de forma ininterrumpida desde mediados de la década de 1990 y aún en curso. Por ello, en esta investigación el análisis se centra en el enfoque y planteamientos del Gobierno en el diseño e implementación de dichas políticas, y en la forma en que el sector del transporte aéreo, en su evolución a lo largo de las últimas dos décadas, impactó positivamente en la conectividad territorial del país, cuyos indicadores se presentan y analizan.

PALABRAS ClAVE | política regional, movilidad, integración territorial.

ABSTRACT | This article analyzes the influence and contribution of air transport to territorial connectivity, using Colombia as a case study. The invaluable contribution of air transport to connectivity between the different regions of the country has been possible thanks to the development of a set of public policies for such industry, which have been implemented uninterruptedly since the mid-1990s and are still in progress. Therefore, the analysis in this research focuses on the approach of the government in the design and implementation of these policies and how the air transport sector, in its evolution in the last two decades, positively impacts the territorial connectivity of the country, which we present and analyze with indicators.

KEYWORDS | regional policy, mobility, territorial integration.

Recibido el 5 de enero de 2019, aprobado el 20 de abril de 2019.

E-mail: oscardiazolariaga@usantotomas.edu.co 


\section{Introducción}

El transporte aéreo desempeña un papel importante en la sociedad globalizada de hoy. Los gobiernos de todo el mundo comprenden cada día más que la conectividad aérea es un activo que mejora la competitividad global de las ciudades, regiones y países, y que su desarrollo disminuye los costos de viaje para los consumidores y las empresas, además de facilitar los contactos y el comercio global. Abundan cada vez más las pruebas respecto de que el incremento de la conectividad aérea estimula la productividad, la inversión extranjera directa, el turismo -entre otros factores- en las diferentes regiones de un país (Burghouwt, 2017). En este contexto, muchos gobiernos formulan políticas públicas de transporte aéreo en el marco de sus industrias (locales), para influir o mejorar los resultados de conectividad a nivel regional y territorial, y lograr una cartera de conectividad que satisfaga mejor las necesidades de la sociedad (van de Vijver, Derudder, Bassens, \& Witlox, 2014).

En las diferentes regiones y países del mundo, la mejora de la conectividad territorial debe su positivo desarrollo a lo largo de las últimas tres décadas al estímulo de la industria de la aviación. Ahora bien, esta industria ha tenido que experimentar una evolución significativa desde sus inicios. Un evento relevante que marcó un hito en su historia fue la Convención de Chicago (diciembre de 1944), a partir de la cual se dio comienzo a la regulación de la aviación civil internacional (Organización de Aviación Civil Internacional [OACI], 2006). Pero la regulación de la industria a nivel mundial a través de políticas muy estrictas, y hasta restrictivas, afectó el mercado de la competencia, influyendo negativamente en el precio de los pasajes, en la demanda y, finalmente, en el flujo de pasajeros (Forsyth, 2006). Un gran cambio en estas materias ocurrió el año 1978, cuando en Estados Unidos se liberalizó el sector aerocomercial, evento que continuó en Europa en la década de 1980 y se extendió luego al resto del mundo, a diferentes velocidades (Belobaba, Odoni, \& Barnhart, 2009). La liberalización de la industria concatenó una serie de eventos relevantes, a saber, intercambio de derechos de tráfico (a través de la firma de convenios bilaterales), privatización de las líneas aéreas de bandera, entrada a los mercados de nuevos operadores aéreos privados -en especial los operadores charter y las líneas aéreas de bajo costo-, acuerdos de cielos abiertos, generación de grandes alianzas entre los operadores aéreos, y finalmente la comercialización y privatización de aeropuertos (Wittmer, Bieger, \& Müller, 2011). Todo ello, que sigue aún su curso, eliminó barreras proteccionistas, propició la competencia, las tarifas se redujeron y se estimuló la demanda, triplicando el tráfico aéreo mundial en las últimas tres décadas (3500 millones de pasajeros transportados en más de 34 millones de vuelos en el año 2015 (Asociación Internacional de Transporte Aéreo [International Air Transport Association, IATA], 2016).

En Colombia comenzó a liberalizarse la industria del transporte aéreo a principios de la década de 1990, proceso en que experimentó todos los eventos antes citados, de nivel mundial, a través de una batería de políticas públicas diseñadas exclusivamente para el sector aéreo, y que aún siguen su curso (Díaz Olariaga, 2016a). En este contexto, la presente investigación analiza la influencia que ha tenido el desarrollo de la industria del transporte aéreo colombiano, a través de sus políticas 
públicas, en la dinámica y evolución de la conectividad territorial en las últimas dos décadas y media. Se presenta oportuno y de interés este análisis de conectividad territorial por varias razones. En primer lugar, debido a la compleja geografía de Colombia: i) atravesada de suroeste a noreste por tres cadenas montańosas de la cordillera de los Andes; ii) posee una región insular en el mar Caribe (archipiélago de San Andrés, Providencia y Santa Catalina) a $775 \mathrm{~km}$ de la costa atlántica colombiana; y iii) el $42,3 \%$ del territorio continental es selva amazónica (zona sureste del país). En segundo lugar, el 76\% de la población del país (con 49,3 millones de habitantes) vive en zonas urbanas (Departamento Administrativo Nacional de Estadística [DANE], 2018). Y, finalmente, la gran deficiencia (en cobertura, capacidad y tecnología) de los sistemas de comunicación terrestre-vial y ferroviario (este último prácticamente inexistente) (Díaz Olariaga \& Carvajal, 2016). En definitiva, todo ello conduce a que el transporte aéreo en Colombia es vital, y con una función más que relevante en la conectividad territorial.

En esta línea, el artículo se organiza de la siguiente manera: en primer lugar, en el marco conceptual, se presenta el desarrollo a nivel internacional del transporte aéreo y su evolución desde el punto de vista de su regulación (este análisis es necesario para comprender las líneas de actuación en el desarrollo de la industria del transporte aéreo en Colombia, que se trata en una sección posterior); a continuación, se analiza la relación conceptual entre transporte aéreo y conectividad; luego se desarrolla en detalle el caso de estudio, presentando la situación de Colombia en el contexto de la investigación (con un fuerte énfasis en el desarrollo de las políticas públicas del transporte aéreo); posteriormente, se muestra y analiza un conjunto de indicadores que reflejan la influencia e impacto del desarrollo de transporte aéreo en la conectividad regional-territorial en Colombia. El trabajo se cierra con las correspondientes conclusiones finales.

\section{Marco conceptual}

\section{La regulación del transporte aéreo internacional}

La industria del transporte aéreo o de la aviación civil (para la presente investigación, ambos conceptos se usarán de forma indistinta) empieza a ser formalmente regulada a partir de la Convención de Chicago de 1944, la cual dio nombre a lo que se conoce como Convenio sobre Aviación Civil Internacional (OACI, 2006). El objetivo de esta convención multinacional, que se celebró en la ciudad de Chicago, Estados Unidos, fue establecer un marco para todos los acuerdos bilaterales y multilaterales futuros en torno al uso de los espacios aéreos controlados por los Estados. El convenio resultante delimitaba, a través de instrumentaciones contenidas en el Derecho Internacional, el establecimiento de las rutas aéreas, y dio origen a las denominadas Libertades del Aire (International Civil Aviation Organization [ICAO], 2004), las cuales ejercen una acción reguladora que norma el libre flujo del tránsito aéreo a través de las fronteras de los Estados. Las Libertades del Aire, también conocidas como Derechos de Tráfico, son el instrumento utilizado por los países para el establecimiento de rutas aéreas internacionales. Las aerolíneas necesitan la 
aprobación de los gobiernos implicados de los diferentes países antes de que puedan viajar dentro o fuera de un país, o incluso volar sobre otro país sin aterrizar (OACI, 2006).

Para la industria aérea, la regulación se consideró necesaria como medio de evitar la competencia destructiva y la inestabilidad del mercado (Papatheodorou, 2002). A nivel nacional (o doméstico), se pensó que la regulación de las tarifas y la capacidad de asientos dotaría de estabilidad al mercado, lo que permitiría a los transportistas con licencia institucional crecer y prosperar, al lograr altos factores de carga y economías de escala sin dilución del rendimiento. Y a nivel internacional, la regulación adoptó la forma de acuerdos bilaterales restrictivos entre países con respecto al intercambio de derechos de tráfico, la designación de líneas aéreas autorizadas a volar entre los dos territorios concernidos, el control de tarifas, la frecuencia y la capacidad.

En un orden diferente, otro de los pilares o componentes básicos de la industria del transporte aéreo también estaba fuertemente regulado. Se trata de los aeropuertos, que eran esencialmente proveedores de servicios pasivos para las aerolíneas (Díaz Olariaga, 2017a). Solían ser propiedad de los Estados en cuyos territorios se encontraban, y eran gestionados u operados por los mismos (esto último ha cambiado, pues hoy en día muchos aeropuertos son operados por empresas privadas). En la mayoría de los casos, sus ingresos dependían casi exclusivamente de fuentes y subsidios aeronáuticos; su política de aranceles solía ser determinada por el gobierno sobre una base ad hoc y las actividades comerciales desempeñaban un papel menor (o casi nulo) (Díaz Olariaga, 2015). Por su parte, los servicios de asistencia en tierra generalmente estaban monopolizados institucionalmente por el operador del aeropuerto o la aerolínea de bandera local (Graham, 2014).

\section{La desregulación del transporte aéreo internacional}

En contraste con los partidarios de la regulación, los defensores de la liberalización de la industria creían que la competencia sería beneficiosa para el transporte aéreo; también reclamaron la privatización de las líneas aéreas nacionales (o de bandera) y la comercialización de los aeropuertos, la intensificación de la competencia en los servicios de asistencia en tierra y, más recientemente, han estado a favor de la eliminación de restricciones en la gestión de los sistemas de reservas o CRS (Computer Reservation Systems) (Graham, Papatheodorou, \& Forsyth, 2008). Entonces, y con base en estos antecedentes, el gobierno de los Estados Unidos desreguló en el año 1978 el mercado aerocomercial (o de las aerolíneas) (Goetz \& Vowles, 2009), y siguió una política activa de liberalización a través de los llamados acuerdos de cielos abiertos a nivel internacional (Crook, 2011). Esta política de liberalización tuvo continuidad en Europa, donde se siguió un proceso gradual, en tres fases, entre 1988 y 1997 (Wittmer, Bieger, \& Müller, 2011); desde entonces, las tarifas, las frecuencias y la capacidad son determinadas libremente por las aerolíneas, aunque la Comisión Europea se reserva el derecho de intervenir en contra de precios predatorios, tarifas excesivas y dumping de asientos (Papatheodorou, 2008). En el contexto latinoamericano, la liberalización del transporte aéreo, iniciada a principios de la década de 1990 , se ha desarrollado a diferentes velocidades en los países de la 
región, principalmente a través de los acuerdos subregionales (o multilaterales), y de forma más aislada a través de acuerdos bilaterales entre países (ICAO, 2003).

Como resultado de la desregulación y liberalización del mercado y el surgimiento de nuevas dinámicas competitivas, el entorno corporativo contemporáneo de las aerolíneas se caracteriza hoy por la coexistencia de, principalmente, tres modelos de negocios. En primer lugar, los operadores tradicionales (líneas aéreas de bandera privatizadas y nuevos operadores privados), conocidos como FSC (Full-Service Carrier), que operan destinos cortos, medios, largos y muy largos. En segundo lugar, los operadores charter, que mantienen sus afiliaciones con el sistema de distribución de viajes (y forman parte de grupos integrados verticalmente). Y finalmente, las denominadas aerolíneas de bajo costo o LCC (Low-Cost Carrier), un producto genuino del proceso de desregulación del mercado, principalmente enfocadas en destinos cortos y medios (casi siempre continentales) y cuyo objetivo de mercado es el recreativo (o turístico). Estas aerolíneas han experimentado un gran auge desde mediados de la década de 1990, inicialmente en Europa y los Estados Unidos, pero cada vez más en todo el mundo (Cook \& Billig, 2017).

En lo que se refiere a las tarifas aéreas, la liberalización del sector condujo a una caída sustancial de los precios y con ello a un fuerte aumento de la demanda, aspecto ampliamente tratado en la literatura científica, tanto para el mercado de los Estados Unidos (Levine, 2006; Morrison \& Winston, 1995; Winston \& Peltzman, 2000), como para el europeo y de otras regiones (Belén Rey, 2003; Burghouwt \& de Wit, 2015; Button, 2001; Dobruszkes, 2009; Thompson, 2002).

\section{Impacto de la desregulación en el sector aeroportuario}

La tradicional propiedad y operación pública de los aeropuertos siempre estuvo enfocada en que existían exclusivamente para ofrecer un servicio público de transporte aéreo. Por lo tanto, las buenas prácticas económico-financieras y la gestión comercial no eran prioritarias. Ahora bien, la nueva situación de liberalización y desregulación de la industria del transporte aéreo motivó e incentivó a los aeropuertos a plantearse una nueva fórmula de operación y gestión, con un enfoque mucho más comercial y orientado al negocio. En definitiva, la década de 1980 podría considerarse como el momento de inicio de la comercialización de los aeropuertos (Czerny, 2013; Graham 2014; Wittmer et al., 2011).

La necesidad, y motivación, de enfocar la gestión del aeropuerto hacia lo comercial y el negocio produjeron varios cambios relevantes. En primer lugar, los aeropuertos empezaron a perder sus fuertes vínculos con sus propietarios, el Fisco de cada Estado. Se flexibilizó la gestión de los aeropuertos a través de la creación de autoridades aeroportuarias públicas, pero más independientes. El objetivo era dar más libertad, e incluso autonomía, al operador del aeropuerto, a fin de que pudiera adoptar las nuevas prácticas de gestión y priorizar actividades antes subvaloradas; entre ellas, la mejora de los ingresos no aeronáuticos, el marketing aeroportuario, la gestión financiera, la evaluación comparativa, el control y aseguramiento de la calidad de servicio, etcétera.

Paso seguido, las privatizaciones de los aeropuertos fueron una realidad desde mediados de la década de 1980. Desde entonces, en un número considerable, 
aeropuertos tanto domésticos como internacionales han sido privatizados, evento que empezó en algunos países desarrollados y, en muy pocos años, se trasladó a los países en vías de desarrollo. En este contexto, la privatización es entendida, principalmente, como la transferencia de la operación desde el sector público al sector privado (Díaz Olariaga, 2017b).

\section{Relación entre conectividad, territorio y transporte aéreo}

En términos generales, puede entenderse la conectividad como una cualidad que surge y se desarrolla a partir de la existencia de vínculos entre objetos y funciones que se interrelacionan. De esta manera, la representación física del concepto abstracto de conectividad es el de una estructura que está conformada por una red de corredores (terrestres, aéreos, marítimos o fluviales) que sirven para movilizar bienes y personas entre distintos puntos del territorio. Las características de esta red dependerán, en primer lugar, de los aspectos físicos o estructurales del territorio donde ella se localiza; es decir, de las dificultades o facilidades que este territorio ofrece al despliegue de dicha red. Asimismo, las características de los flujos, en cuanto a movilidad, volúmenes y tipo de recursos movilizados, son también elementos determinantes de la configuración de la red (Figueroa \& Rozas, 2005). Y finalmente, la conectividad sirve a múltiples propósitos en los distintos ámbitos de la actividad económica y social de un país, siendo los más típicos los siguientes: i) conectividad para facilitar la actividad económica y productiva; ii) conectividad para el desarrollo y la integración social; iii) conectividad geopolítica (ejercicio de la soberanía); y iv) conectividad estratégica (conexión de las regiones y/o territorios de un país con otros países) (Rozas \& Figueroa, 2006).

Y ya entrando en el terreno de la conectividad aérea, muchos gobiernos formulan políticas públicas de transporte aéreo para, entre otros objetivos, mejorar la conectividad entre sus territorios. Los argumentos para que el sector gubernamental emprenda tal tarea son diversos, siendo los más importantes: i) los económicos (desarrollo productivo, generación de empleo y riqueza, etcétera), y ii) los sociopolíticos (integración y cohesión territorial) (Burghouwt, 2017; Burghouwt \& Redondi, 2013). En lo que se refiere al primer argumento, el económico, se estima que el transporte aéreo es un factor habilitante para un desarrollo económico más amplio en una región (van de Vijver, Derudder \& Witlox, 2016). Un análisis de la bibliografía existente en la temática confirma que el transporte aéreo de pasajeros y el empleo (en diferentes sectores industriales) en las regiones urbanas (o metropolitanas) están positivamente vinculados (Alkaabi \& Debbage, 2007; Brueckner, 2003; Button \& Taylor, 2000; Goetz, 1992; Green, 2007; Ivy, Fik, \& Malecki, 1995; Liu, Debbage, \& Blackburn, 2006; Neal, 2012). La razón de estas afirmaciones es la observación de que mejores servicios de transporte aéreo implican una mayor accesibilidad, lo cual alienta a las empresas a ubicarse en una región y estimula la expansión de los negocios existentes (Cooper \& Smith, 2005; Zak \& Getzner, 2014). Al parecer, este incremento en la accesibilidad y conectividad contribuye a positivos resultados de la economía en general ligados a un más alto nivel general de la productividad, y ello gracias a un mayor acceso a otros mercados y mejor dinámica de movimiento de los trabajadores entre regiones (Button, Lall, Stough, \& Trice, 1999; Irwin \& 
Kasarda, 1991; Mukkala \& Tervo, 2013; Neal, 2012; Perovic, 2013). Y finalmente, algunas investigaciones destacan que el empleo en el sector de servicios se deriva de la suposición de que la industria de servicios es más sensible al transporte aéreo de pasajeros que otros sectores de la economía (Bel \& Fageda, 2008; Debbage, 1999; Denstadli, 2004; Faulconbridge, Beaverstock, Derudder, \& Witlox, 2009; Percoco, 2010; van de Vijver, Derudder, Bassens, et al., 2014).

\section{Caso de estudio: transporte aéreo y conectividad en Colombia}

\section{Desarrollo y aplicación de las políticas públicas de transporte aéreo}

El Plan Nacional de Desarrollo, elaborado cada cuatro años por el Departamento Nacional de Planeación (en adelante DNP), es el documento que sirve de base y provee los lineamientos estratégicos de las políticas públicas (incluido el plan de inversiones) formuladas por el Presidente de la República de Colombia a través de su equipo de gobierno. Su elaboración, comunicación, evaluación y seguimiento es responsabilidad directa del DNP.

En los planes nacionales de desarrollo anteriores al año 2002 se definen políticas públicas de inversión en infraestructuras del transporte en general, con un gran énfasis en el transporte por carretera; por ese entonces, no se establecían políticas concretas para el sector del transporte aéreo. El primer Plan Nacional de Desarrollo que estableció una política pública para el sector del transporte aéreo, aunque de forma indirecta e implícita, fue el plan del periodo 2002-2006 (DNP, 2002). En su sección "Infraestructura Estratégica para el Transporte", se afirma que el gobierno nacional dará continuidad a la política de inversiones en infraestructuras, donde se supone están incluidas las del sector aéreo. Luego, el Plan Nacional de Desarrollo 2006-2010 (DNP, 2006) dio un salto sustancial en el establecimiento de una política pública para el transporte aéreo. A la vista de la evolución del movimiento de carga aérea y la creciente participación de esta en el valor del comercio exterior, el gobierno nacional reafirmó la importancia que el transporte aéreo había adquirido en Colombia en los últimos años. Sobre tal base, el Plan fija que las acciones relacionadas con la infraestructura aeroportuaria se deben enfocar hacia el mejoramiento en el sistema de transporte aéreo de carga y pasajeros, a través de la intervención sobre terminales aéreos y pistas en aquellos nodos de importancia estratégica para la competitividad nacional y/o regional. Además, el gobierno afirma que continuará con la implementación de los programas de participación privada para la administración y modernización de infraestructura aeroportuaria y la orientación de las inversiones en este tipo de proyectos, de acuerdo con los volúmenes de tráfico. Asimismo, señala que se elaborarán planes para una articulación eficiente y armónica de los aeropuertos, instrumentos que optimicen su operación en el marco territorial y/o regional del que forman parte. Adicionalmente fija que la Autoridad Pública de Aeronáutica Civil (conocida como Aerocivil) deberá implementar programas orientados a generar recursos financieros provenientes de actividades relacionadas con la prestación del servicio de transporte aéreo, tales como la explotación comercial de los terminales y servicios de aeronavegación (Díaz Olariaga, 2016b). 
Posteriormente, el Plan Nacional de Desarrollo 2010-2014 (DNP, 2010), en consideración al importante crecimiento de la industria y su aportación estratégica a la economía nacional a la fecha (2010), estableció que el gobierno nacional continuaría con la aplicación de políticas para el buen desempeño técnico, legal y financiero de los contratos de concesión aeroportuarios en marcha (ocho a la fecha), y los que en un futuro se celebraran. Adicionalmente, se afirma que el gobierno nacional implementará un programa para mejorar la infraestructura de 17 aeropuertos que representan una operación regular de pasajeros en las capitales de departamento y puntos estratégicos identificados por la Autoridad Pública de Aeronáutica Civil. Se indica que en otros 32 aeropuertos se implementará un programa para mantener las condiciones básicas de operación; y que en aquellas regiones apartadas donde el transporte aéreo se presenta como la mejor (o única) alternativa de comunicación, se adelantarán las labores de mejoramiento y mantenimiento de los aeropuertos regionales, incorporando la variable de riesgo en la definición de las acciones programadas. Se agrega que para atender el crecimiento del transporte aéreo, la Autoridad Pública de Aeronáutica Civil modernizará la infraestructura aeronáutica, como los sistemas de tecnología satelital y los sistemas de comunicación, navegación y vigilancia del espacio aéreo que se establecen en el Plan de Navegación Aérea (PNA-COL) (Aerocivil, 2018b), plan que a su vez está orientado por el Plan Mundial de Navegación Aérea de la Organización de Aviación Civil Internacional, de manera que se promueva, junto con los países vecinos, un sistema integral apropiado para la aviación del futuro.

En cuanto a la política pública de inversión en aeropuertos, los incrementos de la inversión pública en infraestructura del transporte (todos los modos) son el resultado no solo de las mayores tasas de crecimiento económico, sino también del hecho de que para la política pública han cobrado importancia temas como la integración del territorio nacional y el protagonismo de la infraestructura en la conectividad de las regiones y en la competitividad de los productos nacionales. Asimismo, y a pesar de esta dinámica de inversión en infraestructuras del transporte, el gobierno colombiano es consciente de que para satisfacer el crecimiento potencial de la demanda de infraestructura que se podría generar en la próxima década es necesario invertir anualmente más de 3\% del PIB hasta 2020 (DNP, 2014), incluyendo, por supuesto, la parte de la inversión que pueda financiarse mediante concesiones con el sector privado (Díaz Olariaga, 2016b; Yepes, Ramírez, Villar, \& Aguilar, 2013).

Entonces, y en el caso de las infraestructuras de transporte aéreo, la política de concesiones de aeropuertos vino acompañada de una política de inversiones. La inversión pública en infraestructuras de transporte aéreo ha venido creciendo paulatinamente en la última década, hasta llegar a un 14,26\% del total del PIB de transporte en el año 2017, el doble con respecto a dos décadas atrás (MinTransporte, 2018). Por otro lado, la inversión privada en aeropuertos ha evolucionado de acuerdo con la dinámica propia de las concesiones, pasando de inexistente en el ańo 1996, año cero de inicio de las primeras concesiones, a un 20\% como porcentaje del PIB de transporte aéreo en el año 2010 (Fedesarrollo, 2012); el acumulado de inversión privada en el periodo 1997-2013 (que cubre las cuatro generaciones 
de concesiones aeroportuarias) suma aproximadamente 1600 millones de dólares (Díaz Olariaga, 2016b).

\section{Política pública para el sector aeroportuario}

Sobre la gestión de la infraestructura aeroportuaria, Colombia ha seguido la tendencia general en América Latina en cuanto a concesionar la administración de dichas infraestructuras (Díaz Olariaga, 2017b). Este modelo busca liberar al Estado del alto gasto requerido por la infraestructura aeronáutica, proceso regulado en el país por una ley nacional (Ley 80 de 1993, artículo 30). Por otra parte, el Estado nacional, a través de otra norma (DNP, 1994), consagró el proceso de concesiones de aeropuertos mediante un plan de ordenamiento de la infraestructura aeroportuaria. El documento, además de plantear una renovación tecnológica, autorizó el proceso de concesión de los aeropuertos a empresas privadas, y consideró la contraprestación económica para el Estado como único elemento para entregar la concesión. A esta contraprestación se le definió como destino: i) alimentar el fondo de compensación aeronáutica para subsidiar a los aeropuertos no rentables; ii) financiar nuevas inversiones en dichos aeropuertos; y iii) financiar los servicios de tráfico aéreo y seguridad (Díaz Olariaga \& Ávila, 2015). Como resultado, desde mediados de la década de 1990, y en varias fases temporales denominadas generaciones, el sector público colombiano entregó en concesión varios aeropuertos del país -un total de 19 a la fecha, los más grandes e importantes de la red aeroportuaria (Díaz Olariaga \& Pulido, 2019) - con el fin de obtener una mejor administración, modernización y expansión, operación, explotación comercial y mantenimiento de las terminales aéreas de mayor uso. En el marco del objetivo de las concesiones aeroportuarias del país, la empresa, sociedad, consorcio o ente concesionario de los aeropuertos se desempeña únicamente como administrador de los mismos.

La primera generación de concesiones se realizó bajo un modelo de ingresos mínimos garantizados al concesionario, con lo cual este no asumía ningún riesgo. En esta fase se cedieron al concesionario todos los ingresos regulados y no regulados de los aeropuertos, a cambio de una contraprestación fija para el Estado. También se establecía para el concesionario la responsabilidad del manejo, mantenimiento y operación del terminal, pista, rampa, instalaciones aeroportuarias y ayudas audiovisuales de aproximación y zonas accesorias (Díaz Olariaga, 2017b). A partir de la segunda generación de concesiones, concretadas en el periodo 2000-2007, las contraprestaciones para el Estado se distribuyeron entre cargos fijos y cargos variables sobre el ingreso bruto del concesionario. Este cambio en los términos de la privatización de los aeropuertos se hizo necesario debido a que los ingresos que estaba recibiendo el Estado por parte de algunos aeropuertos no eran suficientes para llevar a cabo las inversiones que se tenían previstas. En la tercera generación de concesiones, ańo 2010, se mantuvieron y mejoraron las condiciones de la segunda. No obstante, las diferencias más importantes se dieron en que, por un lado, la determinación del aumento del porcentaje de los ingresos brutos no se hacía sobre ingresos fijos y variables, sino que correspondía a un porcentaje determinado que el concesionario debía pagar al Estado. De otra parte, se definió como obligatorio el cumplimiento del plan maestro y, por tanto, la ejecución de las inversiones a cargo 
y riesgo del concesionario, definiéndose un modelo de ingresos estimados a partir de los cuales se equiparaban los ingresos regulados generados. La cuarta generación de concesiones dio inicio el 30 de diciembre de 2014, con la concesión del Aeropuerto Internacional de Barranquilla-Ernesto Cortissoz. Con posterioridad y finalmente, a mediados de 2017, se concesionó el aeropuerto de la ciudad de Pereira (Díaz Olariaga \& Pulido, 2019).

\section{Política pública en el mercado de los operadores aéreos}

Una de las barreras más importantes de acceso a los mercados es la reglamentación y los controles de rutas, capacidad y tarifas. En lo que refiere al mercado local o doméstico, Colombia ha pasado en los últimos 25 ańos de un esquema proteccionista a otro de libertad vigilada (Díaz Olariaga, 2016b). Ahora bien, aunque la política de acceso al mercado nacional ha sido objeto de una liberalización gradual en ańos recientes, todavía se establece un número máximo de operadores para las rutas de pasajeros, y hasta el año 2012 se mantenía control sobre las tarifas de los tiquetes aéreos, un control que se venía flexibilizando paulatinamente, aunque desde ese año las tarifas aéreas han estado totalmente desreguladas (Díaz Olariaga \& Zea, 2018). El efecto inmediato de la liberalización tarifaria fue la disminución sustancial de las tarifas fijadas por las empresas tanto para los destinos nacionales como para los internacionales, y con ello un automático aumento de la demanda, sobre todo en vuelos y destinos nacionales (Díaz Olariaga \& Ávila, 2015).

Con respecto al mercado internacional, en Latinoamérica las iniciativas de integración y flexibilización del tráfico aéreo han avanzado, impulsadas fundamentalmente por las autoridades aeronáuticas y de comercio. Colombia no es ajena a este panorama de desarrollo mundial, habiendo pasado de un modelo proteccionista de restricciones de acceso y control de tarifas a un enfoque intermedio de apertura gradual, a partir de la década de 1990. Es en los últimos veinte ańos que se cuenta con una extensa relación de acuerdos bilaterales, cuyo objetivo ha sido la armonización de las políticas en el otorgamiento de derechos de tráfico, la regulación de las condiciones de acceso a los mercados y la fluidez del transporte aéreo (Díaz Olariaga, 2016b; Oficina de Regulación Económica [ORE], 2011). A partir del año 2009 se consolidó una política de desregularización total, a través de la promoción de firma de acuerdos que abarcaban desde mayores libertades, hasta los llamados "cielos abiertos" con aquellos países con que se tienen vínculos aéreos para garantizar un mercado plenamente libre, todo ello con el objetivo de avanzar hacia el libre acceso al mercado y de tarifas (ore, 2009).

En otro orden, en Colombia, la entrada al mercado de operadores aéreos privados con un modelo de negocio tradicional, o FSC (Full-Service Carrier), se produjo muy pronto luego de la liberalización del sector (principios de la década de 1990). Pero la entrada al mercado de las líneas aéreas con un modelo de negocio de "bajo costo" fue muchos años posterior a la liberalización. Las tres únicas LCC que operan hoy día en el mercado colombiano son EasyFly (que inició operaciones en 2007), VivaColombia (desde 2012) y finalmente Wingo (con operaciones iniciadas en diciembre de 2016) (Aerocivil, 2018a). 


\section{El papel del transporte aéreo en la conectividad del país}

Se puede afirmar que el transporte aéreo en Colombia prácticamente no tiene competencia (a nivel doméstico) con otros medios de transporte, sobre todo para distancias medias y largas (Díaz Olariaga, Bolívar, Gutiérrez, \& Rico Galeana, 2018). Y esta situación viene reforzada por tres factores determinantes. En primer lugar, la compleja geografía del país (atravesado de suroeste a noreste por tres cadenas montańosas de la cordillera de los Andes). En segundo lugar, existe una gran deficiencia (en cobertura, capacidad y tecnología) de los sistemas de comunicación terrestrevial (no existencia de autopistas de media o alta capacidad); Colombia tiene una deficiencia de 65 mil kilómetros de carreteras, en comparación con indicadores de la región (Latinoamérica) (Yepes, Ramírez, Villar, \& Aguilar, 2013). Y en tercer lugar, una situación similar, negativa, se verifica con el sistema de transporte ferroviario, utilizado solo para el transporte de carga, no para pasajeros (Díaz Olariaga \& Carvajal, 2016); y así y todo, ese sistema de transporte necesita más 4 mil kilómetros de vías férreas (para mejorar el transporte de carga) (Yepes et al., 2013).

En otro orden, Colombia concentra todo su desarrollo poblacional, económico y productivo en la región central, oeste y norte del país (o Andina, Pacífica y Caribe, respectivamente). Por ello, la red aeroportuaria, y sobre todo sus principales aeropuertos, se concentran en dichas regiones (hay aeropuertos en todas las ciudades capitales regionales). En definitiva, la geografía del sistema aeroportuario, y su desarrollo, coinciden y son coherentes con la geografía socioeconómica del país, garantizando así -al menos al nivel de estructura de red- una amplia cobertura territorial (Díaz Olariaga \& Carvajal, 2016).

Finalmente, en lo que se refiere a regiones remotas, aisladas y periféricas, en Colombia existe una región insular, el archipiélago de San Andrés, Providencia y Santa Catalina, al cual solo se puede llegar, desde el territorio continental colombiano, vía aérea. Por otro lado, el país cuenta con regiones y ciudades remotas de nulo acceso vía terrestre, como, por ejemplo, las que se encuentran en la región Amazónica, que en este caso bien podrían designarse como regiones aisladas (ya que solo se puede acceder a ellas vía aérea); y finalmente existen varias regiones periféricas, entendiendo por tal su gran distancia a las principales ciudades del país y también su difícil acceso vía terrestre, debido no solo a la propia y accidentada orografía (o relieve) del país, sino también a la inexistencia de una red principal de carreteras que cubra todo el territorio nacional (Díaz Olariaga \& Carvajal, 2016).

\section{Políticas de conectividad aérea a regiones remotas}

En muchos países del mundo existen regiones cuya situación geográfica (gran distancia respecto de las principales ciudades, insularidad, o difícil o nulo acceso vía terrestre), lleva a que se las denomine como "remotas", "periféricas" y "aisladas". Esta condición de compleja accesibilidad se agrava si las rutas aéreas que conectan dichas regiones no son rentables desde el punto de vista aerocomercial. Entonces, con el fin de garantizar su conectividad interna y la propia cohesión territorial, los países con esta problemática desarrollan políticas públicas ad hoc, que promueven lo que se denomina "servicios aéreos esenciales" (SAE) o también "obligaciones de servicio público” (OSP). En esta línea, se identifican cuatro grupos de políticas públicas 
utilizadas para proporcionar conectividad aérea a regiones remotas (e implementadas ya en varios países): i) políticas basadas en rutas; ii) políticas basadas en los pasajeros; iii) políticas basadas en la compañía aérea; y iv) políticas aeroportuarias (Fageda, Suárez-Alemán, Serebrisky, \& Fioravanti, 2018).

En el caso específico de Colombia, las políticas públicas adoptadas corresponden al tercer grupo antes mencionado, esto es, políticas basadas en la compañía aérea. Es así que, en la actualidad, la empresa pública Satena presta los servicios aéreos esenciales en Colombia, incluidos transporte de pasajeros, de correo y carga, priorizando las regiones menos desarrolladas del país. De esta forma, conecta vía aérea aquellas regiones a las cuales, por cuestiones geográficas, de orden público y de pobreza, no llega ningún otro operador aéreo, garantizando así la conectividad e integración territorial (Aerocivil, 2016). Cabe mencionar que el operador público Satena también opera rutas (domésticas) comercialmente rentables, en directa competencia con operadores privados presentes en el mercado colombiano. En definitiva, en todas las ciudades y regiones de Colombia donde existe un aeropuerto (abierto al tráfico comercial), hay disponibilidad de rutas y frecuencias de conexión con las principales ciudades del país (incluida la capital, Bogotá), operadas por privados, por Satena (operador público) y por servicios de taxi aéreo, y cuya intensidad (nivel de frecuencia) depende de la demanda existente (Aerocivil, 2018c).

\section{Transporte aéreo y posconflicto}

La firma del Acuerdo de Paz entre el gobierno colombiano y las FARC, en noviembre de 2016, ha tenido importante influencia en el crecimiento del turismo (tanto emisor como receptivo), materializada en el incremento en más de $27 \%$ del número de llegadas de turistas extranjeros (vía aérea) desde que dio comienzo el periodo de posconflicto, lo cual es consecuencia principalmente de la mejora de la seguridad y promoción internacional (Menchero, 2018; Ministerio de Comercio, Industria y Turismo [Mincit], 2019).

En el nuevo escenario de posconflicto, el gobierno considera el turismo como un sector económico que posibilita el crecimiento y desarrollo de muchas zonas y regiones de Colombia. Por ello, el Plan Nacional de Desarrollo 2014-2018, en línea con el Plan Sectorial de Turismo "Turismo para la Construcción de la Paz (20142018)”, estructura, para el desarrollo común, varios ejes estratégicos y/o temáticos, entre los cuales se encuentra la conectividad aérea y sus infraestructuras asociadas. Más específicamente, se propone mejorar la calidad de las infraestructuras aeroportuarias en todos aquellos destinos que muestran alto potencial turístico (DNP, 2014).

En otro orden, de entre los lineamientos de política del sector aéreo para la estructuración del Plan Estratégico Aeronáutico 2018-2030 (Aerocivil, 2018c), en su línea de acción "Conectividad y Competitividad", se propone: i) desarrollar una política clara conjunta con el Departamento Nacional de Planeación y el Ministerio de Hacienda para los aeropuertos que prestan Servicios Aéreos Esenciales; ii) desarrollar e integrar la política de turismo y paz del posconflicto; ii) promover, conjuntamente con el Ministerio de Comercio, Industria y Turismo, la conectividad a través de esquemas flexibles, para prestar el servicio de transporte a los territorios 
aislados en las rutas sociales operadas por el operador aéreo público Satena y la flota de aerotaxis.

Finalmente, es necesario mencionar que Naciones Unidas identificó ciertas zonas de Colombia como las de mayor riesgo en el posconflicto; para ser más exactos, elaboró una lista de 126 municipios, en 30 de los cuales (un 24\%) no hay ni aeropuertos ni aeródromos que presten servicios de transporte aéreo. Uno de los objetivos del Plan Estratégico Aeronáutico 2018-2030 es disminuir o anular esa brecha (Aerocivil, 2018c).

Transporte aéreo y políticas sobre intermodalidad, sostenibilidad y medioambiente El Gobierno de Colombia, de la mano de las autoridades sectoriales y entidades expertas, desarrolló el Plan Maestro de Transporte Intermodal (PMTI) 2015-2035 (MinTransporte, 2015), como una visión estratégica del país sobre las necesidades en infraestructura y transporte, que asegure el crecimiento económico y potencie su participación dentro de las dinámicas globales. El pMTi es un compromiso de Estado a largo plazo, que contempla una hoja de ruta de los proyectos de infraestructura intermodal más importantes para el país, seleccionados con base en una rigurosa metodología que responde a la búsqueda de mayor competitividad y accesibilidad, condiciones que le permitirán a Colombia ponerse al día frente a las décadas de atraso que tiene en la materia. El PMTi tiene tres objetivos fundamentales:

- Impulsar el comercio exterior, reduciendo los costos generalizados de transporte en los corredores y transversales existentes y venideros, y en los accesos a las grandes ciudades.

- Impulsar el desarrollo regional, mejorando la calidad de las redes regionales con propósitos de accesibilidad (menor tiempo de llegada a capitales departamentales y/o sistema de ciudades, corredores y transversales).

- Integrar el territorio, aumentando la presencia del Estado a fin de que se reduzca el espacio de actividades ilegales y se acerque a ciudadanos y regiones a los mercados principales y los centros de servicios.

En lo que se refiere a la integración y el papel del transporte aéreo dentro del PMTI, el Gobierno de Colombia seguirá como política la "Visión 2030" del Plan Estratégico Aeronáutico 2018-2030, consistente en movilizar 100 millones de pasajeros/año y duplicar el transporte de carga, en un entorno institucional claro, competitivo, conectado, seguro y sostenible, soportado en una infraestructura renovada, una industria robustecida y un talento humano de excelencia. Por ello, sus ejes temáticos y objetivos estratégicos, algunos de los cuales se mencionan a continuación (DNP, 2019), se constituirán en lineamientos de política enmarcados en el PMTi y en el Plan de Navegación Aérea de Colombia (PNA-COL); a saber:

- Conectividad: para construir redes de servicios de transporte aéreo eficiente que conecten las regiones del país con los principales centros de producción y de consumo nacional y mundial.

- Infraestructura y sostenibilidad ambiental: para lograr que la infraestructura, servicios aeroportuarios, de navegación aérea y la intermodalidad cuenten con 
capacidad y eficiencia, de manera de atender el crecimiento de la demanda del sector en un contexto ambientalmente sostenible, y procurar un aumento en la disponibilidad horaria de los aeropuertos que lo requieran.

En desarrollo de lo anterior, las instituciones públicas competentes impulsarán las políticas, planes, programas y proyectos necesarios para garantizar, en condiciones de intermodalidad, competitividad, resiliencia y sostenibilidad ambiental, el desarrollo oportuno y eficiente de infraestructura aeroportuaria y de plataformas logísticas especializadas. Ello se estructurará en el marco del concepto de aeropuertos inteligentes e infraestructura aeronáutica y aeroportuaria con sus servicios asociados, de tal forma que contribuyan a mejorar la cobertura y calidad del transporte aéreo tanto hacia las zonas aisladas, como entre las distintas regiones del país y conexiones internacionales, con el fin de brindar mayores y mejores condiciones de conectividad (DNP, 2019).

\section{Desarrollo de la conectividad territorial vía aérea}

\section{Desarrollo del tráfico aéreo nacional}

En Colombia, el incremento del tráfico aéreo en conjunto (todos los aeropuertos) y total (sumando pasajeros nacionales e internacionales), fue del $863 \%$ en el periodo 1992-2016 (Aerocivil, 2018a). Ahora bien, aunque esta cifra dice mucho sobre el aumento de pasajeros transportados, en transporte aéreo se utiliza, más frecuentemente, el indicador denominado "pasajeros-kilómetro transportados", o más usado en su acrónimo en inglés, RPK (Revenue Passenger Kilometers), que suministra una medida más exacta del tráfico real, ya que considera la distancia que el pasajero recorre en su viaje. Este indicador ayuda a identificar y dimensionar la cobertura geográfica de un aeropuerto (en distancia hacia sus diferentes destinos). Entonces, en el caso de Colombia, el RPK del sistema aeroportuario ha experimentado un relevante y mantenido crecimiento en la última década, resultado de la entrada de nuevos operadores al mercado (en especial los LCC) y de la incorporación, por parte de las líneas aéreas, tanto de nuevos destinos como de un mayor número de frecuencias a todos los destinos (Díaz Olariaga, 2016a).

La figura 1 muestra la evolución del RPK nacional (o doméstico) del conjunto de aeropuertos de Colombia. El incremento del RPK nacional (que prácticamente se triplicó en la última década) se debe exclusivamente al aumento de la oferta de asientos y frecuencias por parte de los operadores aéreos, ya que la red de destinos (red de aeropuertos) se ha mantenido inalterable en las últimas décadas. Este continuo aumento de la oferta de servicio de transporte aéreo, como respuesta a una creciente demanda, fue posible gracias a la mayor capacidad en infraestructura (y modernización tecnológica) de la red colombiana de aeropuertos, producto a su vez del desarrollo de políticas públicas de inversión (pública y privada) en dicha red, implementadas a lo largo de las últimas dos décadas, como se mencionó con anterioridad. En lo que se refiere al transporte de la carga aérea doméstica, en la figura 2 se muestra su evolución (a nivel Colombia) para los últimos 26 años. Como se aprecia, es un mercado que aún no termina de madurar (Díaz Olariaga \& Ávila, 2015). 
En otro orden, es necesario mencionar que el verdadero motor del crecimiento del tráfico aéreo a nivel nacional fue el Aeropuerto Internacional de Bogotá-El Dorado (воG), en la capital del país, el cual es el principal aeropuerto distribuidor (hub) de la red. BOG conecta con todos los destinos a nivel nacional, es decir, tiene rutas con vuelos programados (o regulares) a todos los aeropuertos del país abiertos al tráfico comercial (Aerocivil, 2018a), lo que implica una cobertura del 100\% en lo que refiere a conectividad aérea a nivel territorial. La figura 3 muestra la evolución de la demanda de pasajes aéreos nacionales (o domésticos) de Bog desde el año 1992 (año siguiente a la liberalización del transporte aéreo en Colombia), y de la misma se puede destacar: i) el crecimiento relevante de la demanda iniciado en el año 2006, cuando se privatizó воG (en cuya ampliación y modernización se invirtieron 650 millones de dólares), potenciado luego por las siguientes generaciones de concesiones aeroportuarias (2008, 2010 y 2015); y una segunda gran ampliación de BOG (entre 2015 y 2018); y ii) la caída de la demanda en el ańo 2017, debido a la huelga de pilotos de la principal aerolínea del país (Avianca) y que duró 51 días (donde la caída de reservas fue de entre el 20 y el 30\%).

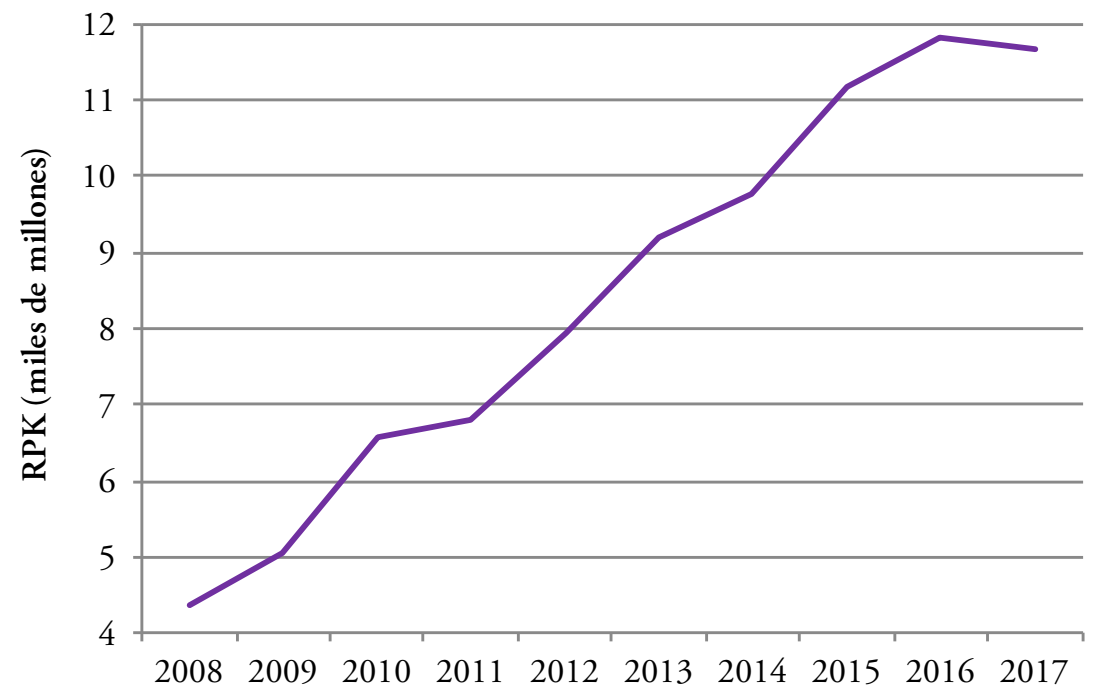

FIGURA I | Pasajeros-kilómetro transportados (RPK) nacional, total Colombia FUENTE: AEROCIVIL, 2OI $8 \mathrm{~A}$ 


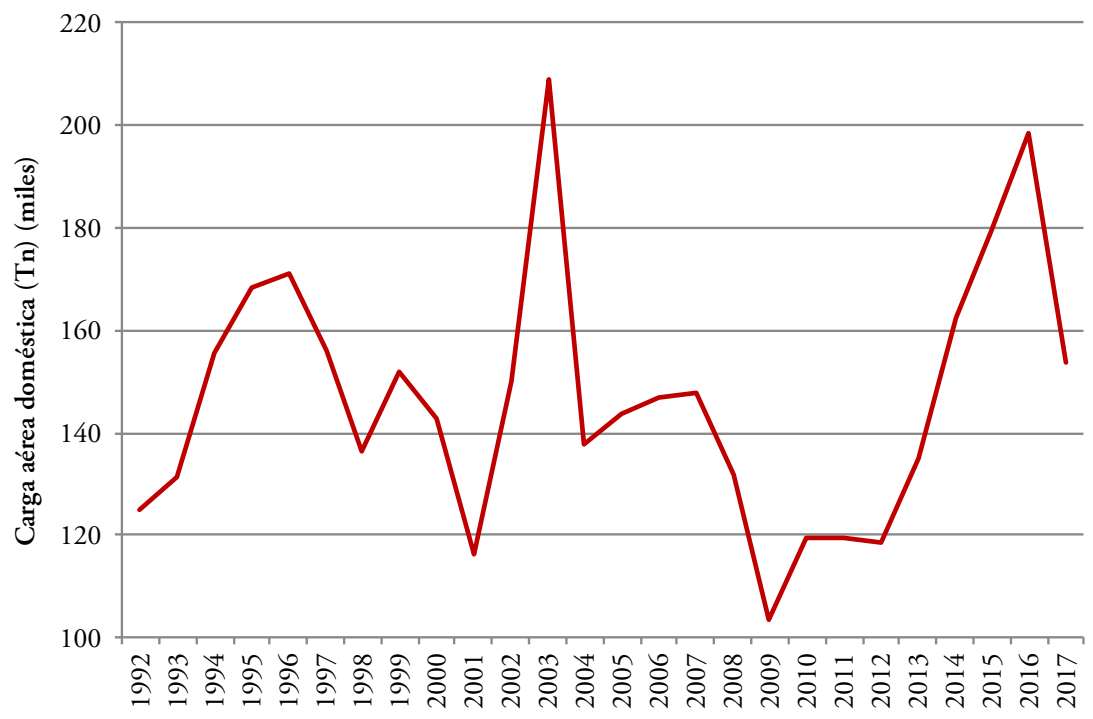

FIGURA 2 Evolución del transporte de carga aérea doméstica (todo Colombia) FUENTE: AEROCIVIL, 2OI $8 \mathrm{~A}$

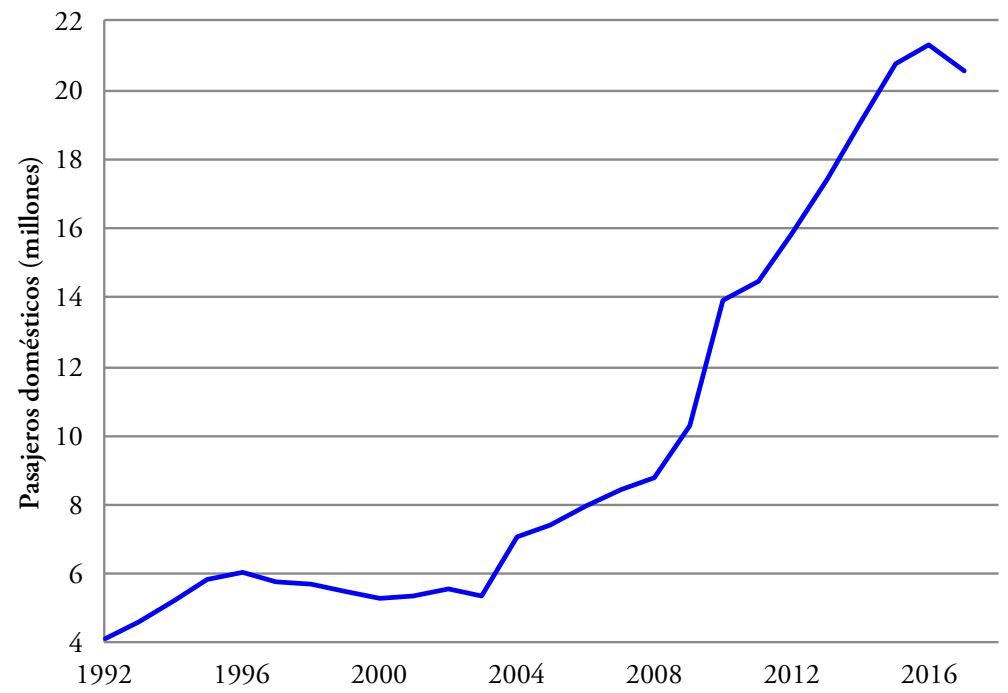

FIgURA 3 | Desarrollo del tráfico doméstico, Aeropuerto Internacional de Bogotá-El Dorado

FUENTE: AEROCIVIL, 2OI $8 \mathrm{~A}$ 
Las previsiones de tráfico para Bog indican que, a mediano plazo (10-15 ańos), el aeropuerto alcanzará su máxima capacidad. Como no existen planes para una nueva (tercera) ampliación (tampoco se contempla esta alternativa, ya que la ciudad ha absorbido al aeropuerto, circunstancia a la que se suman las restricciones ambientales), está en marcha el diseño y construcción de un nuevo aeropuerto internacional. Situado a $15 \mathrm{~km}$ del actual (en las afueras de la ciudad de Bogotá), por ahora conocido como el Dorado II, funcionará simultáneamente con BOG, y se espera que entre en operaciones para el ańo 2026 (Díaz Olariaga, 2017c).

En la tabla 1 se muestran los datos generales de los aeropuertos más importantes del país (y que concentran el grueso del tráfico de la red doméstica colombiana).

\begin{tabular}{|c|c|c|c|}
\hline $\begin{array}{c}\text { CIUDAD DEL } \\
\text { AEROPUERTO }\end{array}$ & CÓDIGO IATA & $\begin{array}{l}\text { GOBERNANZA (AÑo } \\
\text { DE PRIVATIZACIÓN) }\end{array}$ & $\begin{array}{c}\text { PASAJEROS } \\
\text { DOMÉSTICOS }(2017)\end{array}$ \\
\hline Carepa & APO & Privada (2008) & 205.289 \\
\hline Medellín (C) & $\mathrm{EOH}$ & Privada (2008) & 1.070 .441 \\
\hline Rionegro & MDE & Privada (2008) & 6.218 .160 \\
\hline Barranquilla (C) & BAQ & Privada (2015) & 2.327 .531 \\
\hline Cartagena de Indias (C) & СTG & Privada (2010) & 4.052 .383 \\
\hline Valledupar (C) & VUP & Privada (2010) & 388.468 \\
\hline Quibdó (C) & UIB & Privada (2008) & 373.335 \\
\hline Montería (C) & MTR & Privada (2008) & 939.952 \\
\hline Bogotá (D.C.) & BOG & Privada (2007) & 20.582 .580 \\
\hline Riohacha (C) & $\mathrm{RCH}$ & Privada (2010) & 150.393 \\
\hline Santa Marta (C) & SMR & Privada (2010) & 1.691 .473 \\
\hline Cúcuta (C) & CUC & Privada (2010) & 880.329 \\
\hline San Andrés (C) & $\mathrm{ADZ}$ & Privada (2007) & 2.258 .470 \\
\hline Barrancabermeja & EJA & Privada (2010) & 129.313 \\
\hline Bucaramanga $(\mathrm{C})$ & BGA & Privada (2010) & 1.541 .290 \\
\hline Corozal & $\mathrm{CZU}$ & Privada (2008) & 82.687 \\
\hline Cali (C) & CLO & Privada (2000) & 4.265 .459 \\
\hline Pereira* $(\mathrm{C})$ & PEI & Privada (2018) & 1.462 .417 \\
\hline Armenia (C) & AXM & Pública & 377.580 \\
\hline Yopal (C) & EYP & Pública & 338.964 \\
\hline Neiva (C) & NVA & Pública & 328.934 \\
\hline Pasto (C) & PSO & Pública & 287.965 \\
\hline
\end{tabular}

\section{TABLA I Datos generales de aeropuertos principales}

* el ente público adjudicó, en concesión, a finales del año 20i7, el aeropuerto de la CIUDAD DE PEREIRA, PERO EL MISMO SE ENTREGÓ (FORMALMENTE) AL CONCESIONARIO A MEDIADOS DE 2OI8. (C): CIUDAD CAPITAL DE DEPARTAMENTO (NOMBRE DE LA UNIDAD ADMINISTRATIVA REGiONAL EN LAS CUALES SE DiVIDE EL PAÍs); (D.C.): Distrito CAPITAL (CIUDAD CAPITAL DE PAís).

FUENTE: AEROCIVIL, 2OI $8 \mathrm{~A}$ 
En la figura 4 se representa el crecimiento porcentual del tráfico de pasajeros domésticos de los aeropuertos más importantes del país en el periodo 1992-2017. Es necesario destacar que el relevante crecimiento del tráfico aéreo no se verifica solo en el principal aeropuerto del país (y hub), Bogotá-Bog (en el centro geográfico del país), sino que iguales o aún mayores crecimientos de transporte de pasajeros domésticos experimentan las ciudades periféricas de las regiones de la costa Caribe (CZU, SMr, vup, CTG, BAQ), de la costa Pacífico (UIB), insular (ADZ), y de la Andina Central (PEI, AXM, APO, MDE).

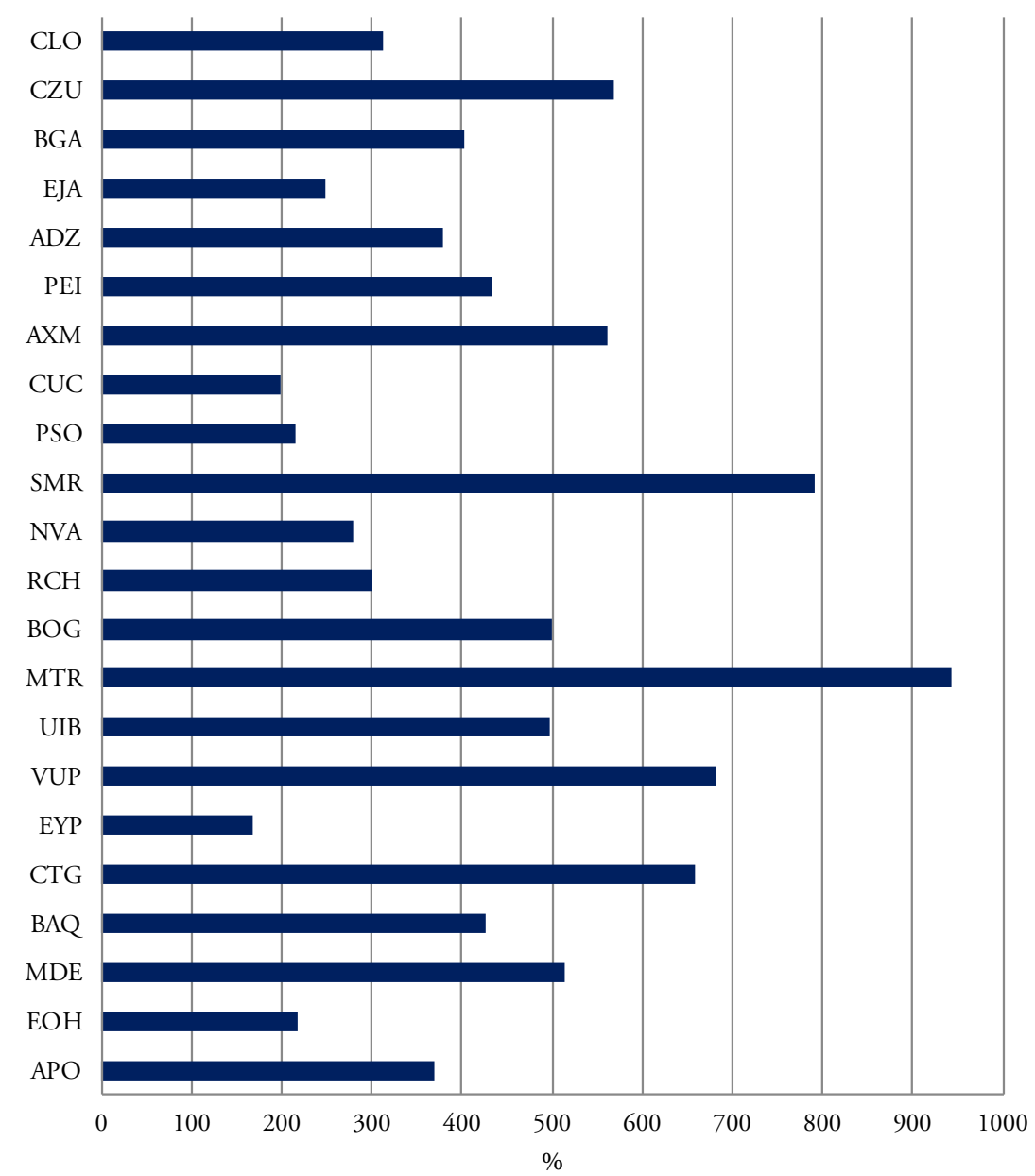

FIGURA 4 | Crecimiento porcentual del tráfico de pasajeros domésticos de los aeropuertos más importante de Colombia, periodo 1992-2017

FUENTE: AEROCIVIL, 2OI $8 \mathrm{~A}$ 


\section{Desarrollo de la conectividad territorial}

La Autoridad Aeronáutica Civil de Colombia, a través de su Plan de Navegación Aérea (Aerocivil, 2018b), estructura sobre el geoespacio aéreo colombiano lo que denomina "áreas homogéneas". Estas áreas, cuatro en total, son grandes corredores aéreos que aglutinan todo el flujo del tráfico aéreo (vuelos), y en los cuales se agrupan todas las rutas de tráfico aéreo. A nivel doméstico, las rutas que gestionan una mayor cantidad de vuelos (y tráfico de pasajeros) se denominan "rutas nacionales principales" (cada ruta conecta dos aeropuertos, y el tráfico en dicha ruta se mide sumando el tráfico en ambas direcciones). Estas rutas principales configuran una verdadera red sobre la geografía colombiana, cuyo desarrollo y crecimiento ha venido reforzado por la propia dinámica del transporte aéreo nacional en las últimas dos décadas. En la figura 5 se presenta el crecimiento de las rutas nacionales principales durante el periodo 1992-2017.

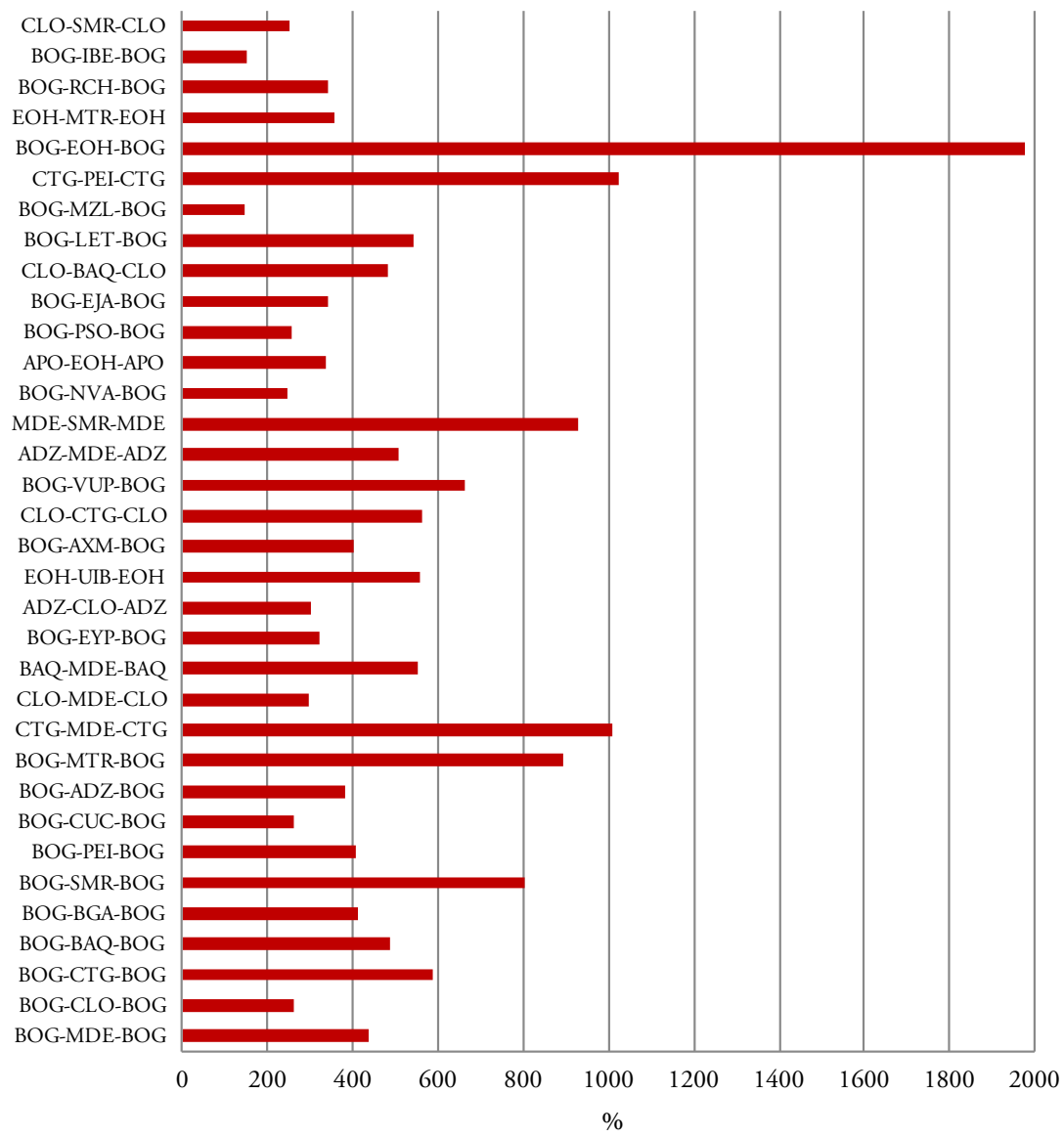

Figura 5 | Crecimiento porcentual del tráfico de pasajeros en las rutas nacionales principales, periodo $1992-2017$

FUENTE: AEROCIVIL, 20I $8 \mathrm{~A}$ 
El indicador mostrado en la figura 5 es el mejor reflejo de la creciente dinámica de la conectividad territorial vía aérea en Colombia, la cual se verifica no solo entre la capital del país (a través de su aeropuerto distribuidor, BOG) y las diferentes regiones, sino que viene aumentando entre las regiones mismas (sin necesidad de conectarse vía la capital del país). Es necesario destacar que los mayores incrementos de tráfico aéreo interregional se verifican entre: i) la capital del país y la región Andina Central (воG-еОн); ii) la capital del país y la costa Caribe (воG-SMr, воG-CTG, воG-MTR, BOG-VUP); iii) la región Andina Central y la costa Caribe (CTG-PEI, MDE-SMr, CTGMDE); y iv) la capital del país y la región Amazónica (BOG-LET).

Los destinos mostrados en la figura 5, a través de los aeropuertos que sirven a dichas ciudades y regiones, representan casi todos los territorios de Colombia, por lo que el crecimiento del tráfico aéreo doméstico ha beneficiado e impulsado, y lo sigue haciendo, la conectividad territorial de país en casi toda su geografía habitada.

\section{Conclusiones}

Como reflejan los indicadores aquí presentados, el gobierno colombiano ha conseguido mejorar la conectividad aérea en sus territorios y regiones, gracias a la implementación, de forma ininterrumpida desde principios de la década de 1990, de un conjunto de políticas públicas (exclusivamente diseñadas para el sector del transporte aéreo).

En la presente investigación se identifica una serie de instrumentos que el gobierno colombiano ha generado e implementado para mejorar la conectividad aérea:

- Liberalización del transporte aéreo.

- Programas de inversión pública en infraestructuras aeroportuarias, para su ampliación y modernización, en todos los aeropuertos de la red, incluso en aquellos de muy reducido tráfico.

- Programa de privatización de aeropuertos.

- Existencia de aeropuertos en todas las regiones (Departamentos) del país (incluida la región insular en el mar Caribe), y varios de los mismos de tráfico internacional.

- Eliminación de barreras para la entrada de nuevos operadores aéreos al mercado.

- Regulación económica de los aeropuertos.

- Desregulación escalonada de las tarifas aéreas, hoy en día totalmente desreguladas.

- Obligaciones de servicio público de transporte aéreo a regiones remotas y/o periféricas donde la operación aerocomercial estándar no es rentable.

- Intercambio de derechos de tráfico con varios países, en especial con los limítrofes, que potencian la conectividad internacional y el transporte de pasajeros y carga de aquellas ciudades y regiones fronterizas del país. 
En lo que se refiere a los efectos socioeconómicos que genera el transporte aéreo y la mejora de la conectividad territorial, la presente investigación no ha podido realizar análisis específicos relacionados (por ejemplo, la influencia en la generación de empleo regional, en el рів de las regiones, etcétera), ya que las instituciones públicas relacionadas en Colombia no generan estadísticas tan específicas (del sector del transporte aéreo) a nivel de regiones y/o municipios. Al respecto, es posible aportar aquí un par de indicadores relacionados de interés. En primer lugar, en el periodo 2000-2014, la aportación del PIB del transporte aéreo al PIB nacional creció de $0,42 \%$ a $0,52 \%$, verificándose un crecimiento medio anual en el periodo del orden del 6\% (DANE, 2018; Díaz Olariaga \& Ávila, 2015).

\section{Referencias bibliográficas}

Aerocivil (2016). Resolución 3442 de 18 de noviembre de 2016 (Diario Oficial n.o 50.085 de 12 de diciembre de 2016). Bogotá: Aerocivil, Gobierno de Colombia.

Aerocivil (2018a). Estadísticas. http://www.aerocivil.gov.co

Aerocivil (2018b). Plan de Navegación Aérea para Colombia. https://bit.ly/3ay0UNO

Aerocivil (2018c). La conectividad aérea en Colombia. Foro Sector Aéreo 2018-2030, 9-10 abril 2018, Bogotá (Colombia).

Alkaabi, K. \& Debbage, K. (2007). Air passenger demand and skilled labor markets by US metropolitan area. Journal of Air Transport Management, 13(3), 121-130. https://doi. org/10.1016/j.jairtraman.2006.11.006

Asociación Internacional de Transporte Aéreo (International Air Transport Association, IATA). (2016). Annual Review 2016. Montreal: IATA. https://bit.ly/30IUZ3G

Bel, G. \& Fageda, X. (2008). Getting there fast: globalization, intercontinental flights and location of headquarters. Journal of Economic Geography, 8(4), 471-495. https://doi. org/10.1093/jeg/lbn017

Belén Rey, M. (2003). Structural changes in the Spanish scheduled flights market as a result of air transport deregulation in Europe. Journal of Air Transport Management, 9(3), 195200. https://doi.org/10.1016/S0969-6997(02)00097-2

Belobaba, P., Odoni, A. \& Barnhart, C. (2009). The global airline industry. Chichester: John Wiley.

Brueckner, J. (2003). Air traffic and urban economic development. Urban Studies, 40(8), 14551469 .

Burghouwt, G. (2017). Influencing air connectivity outcomes. Roundtable on capacity building through efficient use of existing airport infrastructure, 9-10 March 2017, Querétaro.

Burghouwt, G. \& de Wit, J. G. (2015). In the wake of liberalisation: long-term developments in the EU air transport market. Transport Policy, 43, 104-113. https://doi.org/10.1016/j. tranpol.2015.05.006

Burghouwt, G. \& Redondi, R. (2013). Connectivity in air transport networks: an assessment of models and applications. Journal of Transport Economics and Policy, 47(1), 35-53. https://www.jstor.org/stable/24396351 
Button, K. (2001). Deregulation and liberalization of European air transport markets. Innovation: The European Journal of Social Science Research, 14(3), 255-275. https:// doi.org/10.1080/13511610120102619

Button, K., Lall, S., Stough, R., \& Trice, M. (1999). High-technology employment and hub airports. Journal of Air Transport Management, 5(1), 53-59. https://doi.org/10.1016/ S0969-6997(98)00038-6

Button, K. \& Taylor, S. (2000). International air transportation and economic development. Journal of Air Transport Management, 6(4), 209-222. https://doi.org/10.1016/S09696997(00)00015-6

Cook, G. \& Billig, B. (2017). Airline operations and management. New York: Routledge.

Cooper A. \& Smith P. (2005). The economic catalytic effects of air transport in Europe. Final report EEC/SEE/2005/004. Brussel: Eurocontrol.

Crook, R. (2011). U.s. Open Skies agreements number more than one hundred. American Journal of International Law, 105(3), 586-588. https://doi.org/10.1017/S0002930000761677

Czerny, A. (2013). Public versus private airport behavior when concession revenues exist. Economics of Transportation, 2(1), 38-46. https://doi.org/10.1016/j.ecotra.2012.09.001

Debbage, K. (1999). Air transportation and urban-economic restructuring: Competitive advantage in the US Carolinas. Journal of Air Transport Management, 5, 211-221. https://doi.org/10.1016/S0969-6997(99)00015-0

Denstadli, J. (2004). The impact of videoconferences on business travel: the Norwegian experience. Journal of Air Transport Management, 10(6), 371-376. https://doi. org/10.1016/j.jairtraman.2004.06.003

Departamento Administrativo Nacional de Estadística (DANE). (2018). Estadísticas. https:// www.dane.gov.co/index.php/estadisticas-por-tema

Departamento Nacional de Planeación (DNP). (1994). Reordenamiento institucional y plan de expansión del sistema aeroportuario - Documento CONPES 2727. Bogotá: DNP.

Departamento Nacional de Planeación (DNP). (2002). Plan Nacional de Desarrollo 2002-2006. Bogotá: DNP.

Departamento Nacional de Planeación (DNP). (2006). Plan Nacional de Desarrollo 2006-2010. Bogotá: DNP.

Departamento Nacional de Planeación (DNP). (2010). Plan Nacional de Desarrollo 2010-2014. Bogotá: DNP.

Departamento Nacional de Planeación (DNP). (2014). Plan Nacional de Desarrollo 2014-2018. Bogotá: DNP.

Departamento Nacional de Planeación (DNP). (2019). Plan Nacional de Desarrollo 2018-2022. Bogotá: DNP.

Díaz Olariaga, O. (2015). Relevancia actual de los ingresos comerciales aeroportuarios. Estudios Gerenciales, 31(137), 393-402. https://doi.org/10.1016/j.estger.2015.05.003

Díaz Olariaga, O. (2016a). Análisis del desarrollo reciente del transporte aéreo en Colombia. Revista Transporte y Territorio, 14, 122-143. http://dx.doi.org/10.34096\%2Frtt. i1 14.2432

Díaz Olariaga, O. (2016b). Análisis de la evolución de las políticas públicas y de regulación en la industria aeroportuaria en Colombia. Documentos y Aportes en Administración Pública y Gestión Estatal, 26, 7-42. https://doi.org/10.14409/da.v16i26.5934 
Díaz Olariaga, O. (2017a). Análisis de la privatización, regulación y operación aeroportuaria. XII Congreso Colombiano de Transporte y Tránsito. 24-26 de julio de 2017, Bogotá (Colombia).

Díaz Olariaga, O. (2017b). Políticas de privatización de aeropuertos. El caso de Colombia. Documentos y Aportes en Administración Pública y Gestión Estatal, 29, 7-35. https://doi. org/10.14409/da.v17i29.7093

Díaz Olariaga, O. (2017c). Prognosis de tráfico aéreo. El caso del Aeropuerto Intl. de Bogotá (Colombia). Working Paper. https://doi.org/10.13140/RG.2.2.31292.74882

Díaz Olariaga, O. \& Ávila, J. (2015). Evolution of the airport and air transport industry in Colombia and its impact on the economy. Journal of Airline and Airport Management, 5(1), 39-66. http://doi.org/10.3926/jairm.43

Díaz Olariaga, O., Bolívar, N., Gutiérrez, R., \& Rico Galeana, O. (2018). Gravitational analysis of the air transport network. Application to the case of Colombia. Transportation Research Procedia, 33, 51-58. https://doi.org/10.1016/j.trpro.2018.10.075

Díaz Olariaga, O. \& Carvajal, A. F. (2016). Efectos de la liberalización en la geografía del transporte aéreo en Colombia. Cuadernos Geográficos, 55(2), 344-364. https://doi. org/10.30827/cuadgeo.v55i2.3821

Díaz Olariaga, O. \& Pulido, L. (2019). Measurement of airport efficiency. The case of Colombia. Transport and Telecommunication, 20(1), 40-51. https://doi.org/10.2478/ $\mathrm{ttj}-2019-0004$

Díaz Olariaga, O. \& Zea, J. F. (2018). Influence of the liberalization of the air transport industry on configuration of the traffic in the airport network. Transportation Research Procedia, 33, 43-50. https://doi.org/10.1016/j.trpro.2018.10.074

Dobruszkes, F. (2009). Does liberalization of air transport imply increasing competition? Lessons from the European case. Transport Policy, 16(1), 29-39. https://doi. org/10.1016/j.tranpol.2009.02.007

Fageda, X., Suárez-Alemán, A., Serebrisky, T., \& Fioravanti, R. (2018). Air connectivity in remote regions: A comprehensive review of existing transport policies worldwide. Journal of Air Transport Management, 66, 65-75. https://doi.org/10.1016/j. jairtraman.2017.10.008

Faulconbridge, J., Beaverstock, J., Derudder, B., \& Witlox, F. (2009). Corporate ecologies of business travel in professional service firms: Working towards a research agenda. European Urban and Regional Studies, 16(3), 295-308. https://doi. org/10.1177/0969776409104694

Fedesarrollo [Centro de Investigación Económica y Social]. (2012). Infraestructura de Transporte en Colombia: ¿̨luz al final del túnel? $9{ }^{\circ}$ Congreso Nacional de la Infraestructura, 21-23 noviembre 2012, Cartagena de Indias.

Figueroa, O. \& Rozas, P. (2005). Conectividad, ámbitos de impacto y desarrollo territorial: el caso de Chile. Santiago de Chile: Comisión Económica para América Latina y el Caribe (Cepal).

Forsyth, P. (2006). Martin Kunz Memorial Lecture. Tourism benefits and aviation policy. Journal of Air Transport Management, 12, 3-13. https://doi.org/10.1016/j. jairtraman.2005.09.001 
Goetz A. (1992). Air passenger transportation and growth in the U.s. urban system, 19501987. Growth and Change, 23, 218-242. https://doi.org/10.1111/j.1468-2257.1992. tb00580.x

Goetz, A. \& Vowles T. (2009). The good, the bad, and the ugly: 30 years of US airline deregulation. Journal of Transport Geography, 17(4), 251-263. https://doi. org/10.1016/j.jtrangeo.2009.02.012

Graham, A. (2014). Managing Airports: An International Perspective. London: Routledge.

Graham, A., Papatheodorou, A., \& Forsyth, P. (eds.). (2008). Aviation and tourism: Implications for leisure travel. Burlington: Ashgate.

Green, R. (2007). Airports and economic development. Real Estate Economics, 35(1), 91-112. IATA. Véase Asociación Internacional de Transporte Aéreo.

ICAO. Véase International Civil Aviation Organization.

International Civil Aviation Organization (ICAO). (2003). Background of Liberalization and experiences in the Latin American Region. Worldwide Air Transport Conference ATConfl5-WP/98, 24-29 March 2003, Montreal.

International Civil Aviation Organization (ICAO). (2004). Manual on the Regulation of International Air Transport. Montreal: ICAO.

Irwin, M. \& Kasarda, J. (1991) Air passenger linkages and employment growth in U.s. metropolitan areas. American Sociological Review, 56(4), 524-537. https://doi. org/10.2307/2096272

Ivy, R., Fik, T., \& Malecki, E. (1995). Changes in air service connectivity and employment. Environment and Planning, 27, 165-179. https://doi.org/10.1068/a270165

Levine, M. (2006). Why weren't the airlines reregulated? Yale Journal on Regulation, 23(2), 269297. https://digitalcommons.law.yale.edu/yjreg/vol23/iss $2 / 5$

Liu, Z., Debbage, K., \& Blackburn, B. (2006). Locational determinants of major US air passenger markets by metropolitan area. Journal of Air Transport Management, 12, 331-341. https://doi.org/10.1016/j.jairtraman.2006.08.001

Menchero, M. (2018). Colombia en posconflicto: ¿turismo para la paz o paz para el turismo? Araucaria, Revista Iberoamericana de Filosofí, Política y Humanidades, 20(39), 415438. https://revistascientificas.us.es/index.php/araucaria/article/view/4914

Ministerio de Comercio, Industria y Turismo, Colombia (Minciт). (2019). Informes de Turismo. http://www.mincit.gov.co/publicaciones/16590/informes_de_turismo

Ministerio de Transporte, Colombia (MinTransporte). (2015). Plan Maestro de Transporte Intermodal 2015-2035. Bogotá: MinTransporte.

Ministerio de Transporte, Colombia (MinTransporte). (2018). Transporte en cifras. Estadísticas 2017. Bogotá: MinTransporte.

Morrison, S. \& Winston, C. (1995). The evolution of the airline industry. Washington, DC: Brookings Institution.

Mukkala, K. \& Tervo, H. (2013). Air transportation and regional growth: which way does the causality run? Environment and Planning, 45(6), 1508-1520. https://doi.org/10.1068/ a 45298

Neal, Z. (2012). Creative employment and jet set cities: disentangling causal effects. Urban Studies, 49, 2693-2709. https://doi.org/10.1177/0042098011431282

Oficina de Regulación Económica (ORE), Colombia. (2009). Recomendaciones sobre lineamientos de política aerocomercial internacional de pasajeros y carga. Bogotá: ORE. 
Oficina de Regulación Económica (ORE), Colombia. (2011). Seguimiento y evaluación de impacto de la política aerocomercial internacional de pasajeros y carga. Bogotá: ORE.

Organización de Aviación Civil Internacional (OACI). (2006). Convenio sobre Aviación Civil Internacional. Montreal: OACI.

Papatheodorou, A. (2002). Civil aviation regimes and leisure tourism in Europe. Journal of Air Transport Management, 8(6), 381-388. https://doi.org/10.1016/S09696997(02)00019-4

Papatheodorou, A. (2008). The impact of civil aviation regimes on leisure travel. En A. Graham, A. Papatheodorou \& P. Forsyth (eds.), Aviation and tourism. Implications for leisure travel (pp. 49-57). Burlington, uK: Ashgate.

Percoco, M. (2010). Airport activity and local development: Evidence from Italy. Urban Studies, 47(11), 2427-2443. https://doi.org/10.1177/0042098009357966

Perovic, J. (2013). The economic benefits of aviation and performance in the travel \& tourism competitiveness index. En J. Blanke \& T. Chiesa (eds.), The travel \& tourism competitiveness report. Reducing barriers to economic growth and job creation (pp. 57-61). Genève: World Economic Forum.

Rozas, P. \& Figueroa, O. (2006). Conectividad, ámbitos de impacto y desarrollo territorial: análisis de experiencias internacionales. Santiago de Chile: Comisión Económica para América Latina y el Caribe (Cepal). https://repositorio.cepal.org/bitstream/ handle/11362/6314/1/S0600566_es.pdf

Thompson, I. (2002). Air transport liberalization and the development of third level airports in France. Journal of Transport Geography, 10(4), 273-285. https://doi.org/10.1016/ S0966-6923(02)00043-1

van de Vijver, E., Derudder, B., Bassens, D., \& Witlox, F. (2014). Filling some black holes: Modeling the connection between urbanization, infrastructure, and global service intensity. The Professional Geographer, 66(1), 82-91. https://doi.org/10.1080/003301 24.2013.781488

van de Vijver, E., Derudder, B., \& Witlox, F. (2016). Air passenger transport and regional development: cause and effect in Europe. Promet - Traffic \& Transportation, 28(2), 143-154. https://doi.org/10.7307/ptt.v28i2.1756

Winston, C. \& Peltzman, S. (2000). Deregulation of network industries: what's next? Washington, DC: AEI Brookings Joint Center for Regulatory Studies.

Wittmer, A., Bieger, T., \& Müller, R. (2011). Aviation Systems. Heidelberg: Springer.

Yepes, T., Ramírez, J., Villar, L., \& Aguilar, J. (2013). Infraestructura de transporte en Colombia. Cuadernos Fedesarrollo 46. Bogotá: Fedesarrollo.

Zak, D. \& Getzner, M. (2014). Economic effects of airports in Central Europe: A critical review of empirical studies and their methodological assumptions. Advances in Economics and Business, 2(2), 100-111. https://doi.org/10.13189/aeb.2014.020206 